\title{
Prediction of Volleyball Competition Using Machine Learning and Edge Intelligence
}

\author{
Qiang Liu and Qiannan Liu \\ The Graduate School, Woosuk University, Wanju, Republic of Korea \\ Correspondence should be addressed to Qiannan Liu; emma@hgu.edu.cn
}

Received 17 January 2021; Revised 7 February 2021; Accepted 27 February 2021; Published 8 March 2021

Academic Editor: Muhammad Babar

Copyright ( 2021 Qiang Liu and Qiannan Liu. This is an open access article distributed under the Creative Commons Attribution License, which permits unrestricted use, distribution, and reproduction in any medium, provided the original work is properly cited.

\begin{abstract}
Data analysis and machine learning are the backbones of the current era. Human society has entered machine learning and data science that increases the data capacity. It has been widely acknowledged that not only does the number of information increase exponentially, but also the way of human information management and processing is completed to be changed from manual to computer, mainly depending on the transformation of information technology including a computer, network, and communication. This paper is aimed at a solution to the lag of the methods and means of volleyball technique prediction in China. Through field visits, it is found that the way of analysis and research of techniques and tactics in Chinese volleyball practice is relatively backward, which to a certain extent affected the rapid development of Chinese volleyball. Therefore, it is a necessary and urgent task to realize the reform of the methods and means of volleyball technical and tactical analysis in China. The data analysis and prediction are based on the machine learning and data mining algorithm applied to volleyball in this paper is an inevitable trend. The proposed model is applied to the data produced at the edges of the systems and thoroughly analyzed. The Apriori algorithm of the machine learning algorithm is utilized to process the data and provide a prediction about the strategies of a volleyball match. The Apriori algorithm of machine learning is also optimized to perform better data analysis. The effectiveness of the proposed model is also highlighted.
\end{abstract}

\section{Introduction}

The development of the modern Olympic Games for more than 100 years has proved that the improvement of the performance of competitive sports has gone through the following four stages in turn. Machine learning is widely used to predict sports [1]: the period of natural growth, the period of a large amount of movement, the period of high-intensity growth, and the period of scientific growth [2]. A detailed review is also provided in this context [3]. At present, competitive sports are in the fourth stage when its remarkable feature is that science and technology have been involved in the whole process of competitive sports from material selection, training, and competition to recovery. In other words, science and technology are the wings of competitive sports [4]. Now the human society has entered the IT Era when the marked sign is that not only does the number of information increase exponentially but the way of information management and processing relies mainly on information technology including a computer, network, and communication. In other words, the level of information collection, management, processing, and analysis is the determinant of the correctness of human decision-making and behavior selection [5].

It is reported that Liu Xiang, a Chinese track and field athlete who won the gold medal in the 110-meter hurdles at Athens Olympic Games, has more than 70 scientific research institutes and sports companies at home and abroad. They did scientific research for him in the design of running shoes, nutrition supply, recovery and rest, tactical analysis, and other aspects. Only during the preliminaries and finals, there were more than 10 scientific research personnel of the National Sports Administration Institute, Shanghai Sports Scientific Research Institute, and CCTV analyzing and comparing the hurdles and tactics of the athletes Liu Xiang 
and Alan Johnson in both Beijing and Athens by modern computer information means, such as video acquisition, comparison on the same screen, the transmission of streaming media network, and so on. Also, this staff provided feedback of the information to Liu Xiang in time, which made him know himself as well as the enemy and make the best use of the advantages and bypass the disadvantages. Liu Xiang played his competitive level normally and even supernormally in the game and contributed to the historical breakthrough of Chinese track and field [6].

Data mining technology and tactics statistics of sports are excavated through two technologies, data mining and correlation analysis and visualization [7]. The use of the volleyball technique and tactics analysis model (BTT) is applicable not only to precompetition preparation and postgame summary, but also to the analysis of technical and tactical data in the game $[8,9]$. In the field of data mining, this study attempts to use association analysis and visualization technology to make a preliminary excavation of the information behind the volleyball technical and tactical data. As far as the breadth of mining means and the depth of the mining content are concerned, it is far from enough, so these deficiencies will be the direction and task of the follow-up study. The Apriori technique is used for the recurrent element set mining [10]. It is also based on the learning of the association rule over relational Database Management systems (DBMS). It operates by recognizing the recurrent distinct objects in the DBMS and outspreading them to bigger and bigger object sets until necessarily repeated in the DBMS.

\section{Literature Review}

Machine learning methods and tactics statistics of volleyball can be mined through two types of machinery, which are data mining and correlation analysis and visualization [11]. The usage of the volleyball practice and strategies investigation model is appropriate for precompetition preparation and postgame summary [12]. It can also be utilized to analyze the technical and tactical data during the game. In the field of data mining, this study attempts to use association analysis and visualization technology to make a preliminary excavation of the information behind the volleyball technical and tactical data [13]. As far as the breadth of mining means and the depth of the mining content are concerned, it is far from enough, so these deficiencies will be the direction and task of the follow-up study. The Apriori technique is used for the recurrent element set mining [14]. It is also based on the learning of the association rule over relational Database Management systems (DBMS). It operates by recognizing the recurrent distinct objects in the DBMS and outspreading them to bigger and bigger object sets until necessarily repeated in the DBMS.

For many years, scholars at home and abroad have carried out the substantive study of volleyball techniques and tactics at the same time along several different paths. The scholars use the logical method of history to find the time and background of the appearance of various volleyball techniques, such as variable direction dribble appearing in
1908, volleyball hook shot appearing in 1940, and so on [15]. The scholars have put forward a new point of view through the analysis of kinematics and dynamics of volleyball technique by using biomechanics; with the help of highspeed photographic instruments, the athletes' technical movements are photographed, with three-dimensional kinematics and dynamics analysis of the film or simplified processing of motion technology to build a model for mechanical analysis. Also, using kinematics, classical mechanics, anatomy, and even aerodynamics knowledge and principles, scholars have made some useful discussions on the posture, aiming point, and rotation of the ball. For example, the direction of the arm and the finger should be close to the middle axis of the human body.

Association rule mining is largely apprehensive with unseen, motivating, and valued relationships among relational databases (RDMS), transaction databases, and massive data items [16]. These huge and massive data items are in the form of information storage, which is one of the important research contents in data mining. Among them, the Apriori algorithm is the most classical association rule extraction algorithm. The hollow ball cast aiming point should be selected in the net rather than the traditional textbook advocated distal proximal, and the ball in the air should spin, which is conducive to high arc [17]. Scholars also put forward a study of volleyball technology from the perspective of psychology. For example, the use of positive self-hints in a free throw can help the completion of a technical movement. In volleyball training, the imagination training method, the image training method, the simulated competition scene training method, and the self-confidence training method are used [18]. There are several proposals that are focusing on sports prediction [19-21].

Edge computing is carried out to improve the processing of the systems. Edge computing is a viewpoint of computer networking that is based on the idea of carrying processing as close to the data. It improves the processing. The Apriori method of machine learning can be applied to edge computing to perform faster processing. The traditional data analysis can also be improved by utilizing the machine learning mechanism. The Apriori method is one of the widely used methods to perform data analysis. The processing activity can also be improved if the data computation is done using the edge computing concept. The traditional Apriori has several challenges including the bottleneck challenge. Therefore, there is a need for optimization of this algorithm to solve the bottleneck problem of the Apriori algorithm and improve the efficiency of the algorithm.

\section{Proposed Methodology}

This section describes the proposed methodology for the prediction of volleyball matches using edge-enabled data analysis. The proposed model is based on a machine learning algorithm. The classical Apriori algorithm of machine learning is utilized in this research. The classical Apriori algorithms are extended and optimized to overcome the challenges faced by the traditional approach. The Apriori technique is used for the recurrent element set mining. It is 
also based on the learning of the association rule over relational database management systems (DBMS). It operates by recognizing the recurrent distinct objects in the DBMS and outspreading them to bigger and bigger object sets until necessarily repeated in the DBMS. The detailed description of the proposed extended Apriori algorithms is shown below.

3.1. Extended Apriori Algorithm. Association rule mining is mainly concerned with hidden, interesting, and valuable laws between relational databases, transaction databases, and massive data items in information storage, which is one of the important research contents in data mining. Among them, the Apriori algorithm is the most classical association rule extraction algorithm, and other related algorithms are improved or extended based on the algorithm. To further study the algorithm of association rule mining, we first introduce the classic Apriori algorithm. The Apriori algorithm uses an iterative method of layer-by-layer exploration, and the $k$-term set is used to find the $(k+1)$-itemset. First, the frequent 1-term set $\mathrm{Li}$ is obtained, and the frequent 2term set $L 2$ is searched with $\mathrm{Li}$, and $L 2$ is used to search for $L 3$, and so on, until the frequent $(k+1)$-itemset cannot be found. Among them, the largest frequent itemsets are $k$ frequent itemsets, and each $L k$ needs to scan a database once. The nature of Apriori: the nonempty subset of any frequent itemset is also a frequent itemset. The nature of Apriori is used to search for frequent itemsets with two steps: connection and pruning. In the connection part, the $\mathrm{Lk}-i$ is connected with the $\mathrm{Lk}-i$ to generate a possible candidate itemset; in the pruning part, the candidate itemsets with infrequent subsets are deleted by using the Apriori property.

From the description of the above algorithms, it is not difficult to find that although the Apriori algorithm reduces the number of candidate items, in the execution of the "connection-pruning" iteration it is still inevitable to test a large number of candidate sets frequently and to scan the database repeatedly. When the database is large enough to repeatedly scan the external needs, the efficiency is low. Processing $\mathrm{I} / \mathrm{O}$ operations for large-scale data has become a bottleneck for improving efficiency. Therefore, to improve the efficiency of the Apriori algorithm, the defects of the Apriori algorithm will be improved in the next chapter. A matrix candidate itemset and frequent itemsets are proposed. To reduce the time overhead of the corresponding operation, the matrix is compressed according to the related properties.

The set item $F_{q}^{\prime}$ : the result attribute in the matching relational database is the case of $R_{k}$ and the attribute value is $d$; if the item $R_{k}(d \longrightarrow \sim d)$ is expressed as $F_{q}^{m}$, then $r^{m}: F_{1}^{m} \wedge F_{2}^{m} \wedge \ldots \wedge F_{L^{\prime}}^{m} \Rightarrow F_{q}^{m} . F_{i}^{\prime}$ belongs to the state before the change, and $F_{i}^{\prime \prime}$ belongs to the state that has changed. In this case, the support degree sup $(R)$, lifting quantity lift-measure $(R)$, and confidence conf $(R)$ of rule $R$ are as follows:

$$
\begin{aligned}
\sup (r) & =\sup \left(r^{\prime}\right), \\
\operatorname{lift\_ measure}(r) & =\operatorname{lift}\left(r^{\prime}\right)-\operatorname{lift}\left(r^{\prime \prime}\right), \\
\operatorname{con}(r) & =\frac{\operatorname{lift}\left(r^{\prime}\right)-\operatorname{lift}\left(r^{\prime \prime}\right)}{\operatorname{lift}\left(r^{\prime}\right)}=\frac{\operatorname{lift\_ measure}(r)}{\operatorname{lift}\left(r^{\prime}\right)} .
\end{aligned}
$$

The degree of support in the rule represents the scope of influence, which shows the usefulness of the rules. Lifting capacity showed the degree of change, enhancing the degree of higher representatives will be the value of the $F_{i}$ property from $a_{i}$ to $a_{j}$; the $R_{k}$ property value is $d$ in reducing the more. Confidence is the certainty that shows the change. Then for the nature of lift_measure, for association rules, the lifting degree belongs to a class of relative low complexity measures. The formula for calculating the lifting degree between $A$ and $B$ is

$$
\text { lift }(A, B)=\frac{P(A \cup B)}{P(A) P(B)} .
$$

By the expression of lift in the association rules, the correlation metric formula based on the lifting quantity can be expressed as

$$
\operatorname{corr}_{(A 1 \longrightarrow A 2) \cdot(B \longrightarrow \sim B)}=\operatorname{lift}(A 1, B)-\operatorname{lift}(A 2, B) .
$$

It is important to note that, in the form, $A 1$ represents the set of initial sets, and $A 2$ represents the set of itemsets for $A 1$ mutex, and $B$ represents the set of the result. Here we contact to reduce the occurrence of $B$. The proposed extended algorithm is also optimized to effectively utilize and achieve better results. The algorithm efficiency is improved in the context of bottleneck challenges.

3.2. Algorithm Optimization. The proposed algorithm is optimized to solve the bottleneck problem of the Apriori algorithm and improve the efficiency of the algorithm. This research improves the efficiency in the context of the few concepts. First, the source database is scanned, based on the source transaction database, and the transaction matrix $D$ is constructed according to the definition. The transaction is the line and the itemset is the column when the transaction matrix is constructed. In order to facilitate the compression of a matrix, a column of sum_c is added to the matrix to record the number of items contained by each firm. And a row of sum $r$ is added to the matrix to record the count of each item's support. According to the definition, the minimum support transaction number min_supsh is calculated and the matrix is compared. If the value of an element is less than min_supsh, the column corresponding to the element is deleted, from which we get frequent 1-term sets. The sum_c column is recalculated; if the value of an element is 0 , the line 
of the element is deleted. Thus, the matrix $D 1$ is produced. When compressing the matrix $D 1, K$-frequent itemset $(k \geq 2)$ is produced. According to the property 1 , the column corresponding to the item $\mathrm{Ij}$ of the frequent $(k-1)$-itemset less than $k$ is deleted. The sum_c column is recalculated again, and then according to the property 2 , if the value of an element is less than $k$, the element corresponding to the element is deleted. Recalculate the sum_r line; if the value of an element is less than min_supsh, the column corresponding to the element is deleted. The matrix is compressed repeatedly until it can not be compacted again. The matrix $\mathrm{Dk}$ after the compression is obtained.

Set up an auxiliary two-dimensional array, which is used to record the frequency and the corresponding combination of subscript mix, so you can more easily find frequent itemsets $k$ - $(k \geq 2)$. According to definition 3 , we collected the column vector of the matrix $\mathrm{Dk}$ corresponding $K$ dimensional combination and for each combination of vector and bitwise operations on a K column and for "number 1" and to obtain the $K$ dimension of the support number. If the support number of $K$ dimension is not less than min_supsh, then the itemset in the combination pair is a frequent $k$-itemset. The results are stored in a two-dimensional array, the front is the subscript of the corresponding set of items, and the last column is the itemset to support the number of transactions. According to the nature, if the number of frequent $k$-itemsets is less than $k+1$, the search frequent itemsets are terminated. Otherwise, repeat the above call until the generated frequent itemsets are empty. This process improves the efficiency of the proposed extended Apriori algorithms.

\section{Result and Discussion}

This section describes the results of the simulation. The results are generated using the proposed extended and optimized machine learning algorithm. The dataset is achieved using the edge computing concepts. As mentioned above, although volleyball is a collective event, the one or two players, especially the core players or role players, are not the decisive players, but not all players, because the volleyball match is the winning or losing of the score on both sides of the game, and the precondition of hitting the ball is the right to have the ball. In the game, there are five players on one side, and there is only one volleyball, so the chance of each player is unbalanced in terms of the right of the ball. Volleyball is controlled and disposed by core players in most of the time and crucial moments, and the tactics of the team are also designed and run around core players and role players. So the core and role players of a team, as well as the restrictions on the core and role players of the team, are the key to winning the game. So as long as the analysis of the team's core players and the main players is almost equal to the analysis of a team, the strategic line map of the core players and the main players is shown in Figure 1.

For the high level volleyball players, their technical and tactical patterns are in a dynamic pattern, and the characteristics of attack and defense are relatively fixed. For example, the choice of the way of attacking the ball and the

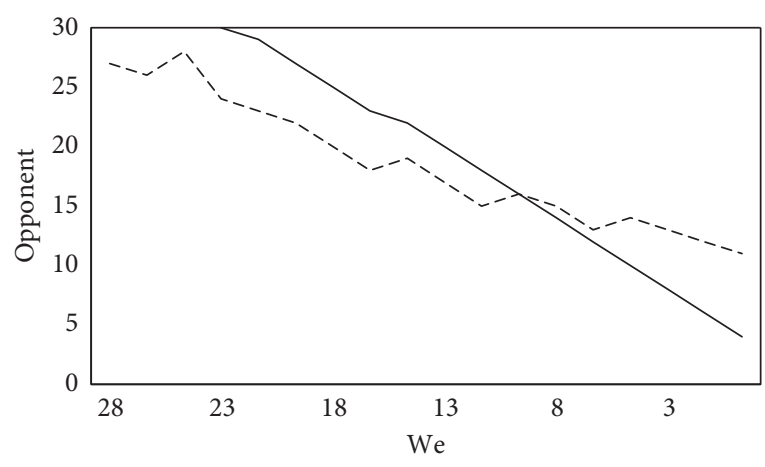

FIGURE 1: Core player and main player strategic line.

area, the defensive position, and the choice of the tactics have certain rules, which provides the possibility for the analysis of tactics and tactics. As shown in Figure 2, the gray part of the picture shows that Liu Wei's free throw score in the first 19 rounds of the CBA League 2005 to 2006 season accounts for the percentage of his total score.

The stability of step point affects the pace of running and the full degree of take-off. In this paper, the difference between the 6 take-off steps is analyzed by Liu Wei (whose running number is 23 steps) as an example. The step position statistics is shown in Table 1. The data unit in the table is meter. It can be seen from Table 1 that the repeatability of the 6 training steps of the athletes is poor, and the accuracy of the pedal is low.

Oksa is the core of Shanghai attacks and defense blocking net score. Therefore, the use of good oksa has become the key to the success of the development of East Shanghai. Here is oksa as the research object, using the time efficient association rules of the competitive ability of technical and tactical data mining. The sources of data are as follows: the video data in the CBA League oksa in 2005 2006 season before the 19 rounds of the competition are gotten by setting game time to query and extract multimedia database of volleyball technique and tactics. We counted the total score of three points, scoring three points, hitting number, three hits and two points, hitting number two and two free throws, hit rate, free throws, free throw percentage, backcourt number, front to back ball number, number, number of backcourt steals, cover number, error number, number of fouls assists, number, number, number of effective defensive tactics in the technical and tactical indicators in 6 minutes, 7 minutes, 8 minutes, 9 minutes, 10 minutes, 11 minutes, and 12 minutes.

We only study the total score and the score two points and two free throws along with the hit rate of free throw percentage. In addition, the offensive backcourt number, stealing the ball back cover number, number of fouls, failure assists, and effective defensive tactics are also taken into consideration. The number of technical and tactical indicators is focused on too. According to the player's tactical competitive ability efficiency formula, division between the corresponding values was weighted and this season has been the game and getting a coefficient. The set of values is as follows: the player's continuous playing time (minutes per minute, its TID value is 


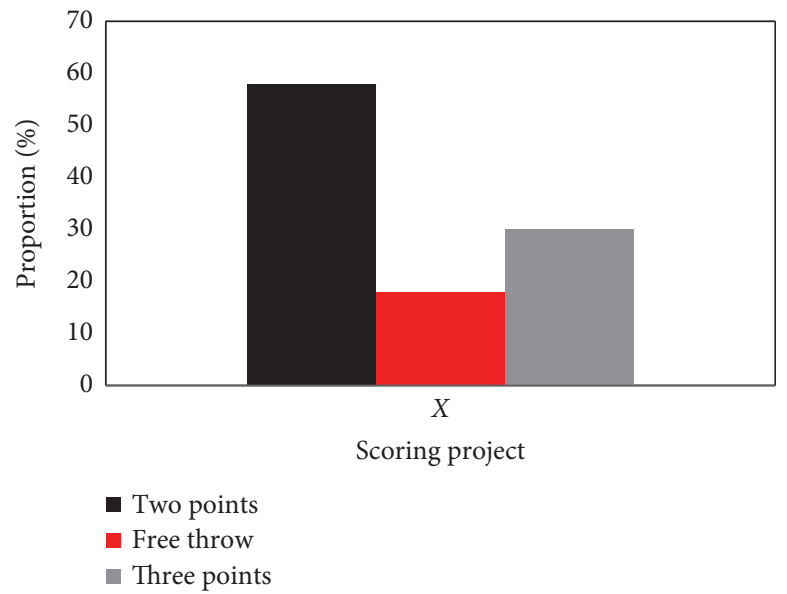

FIgURe 2: Liu Wei's shooting.

TABLe 1: Statistics of the full range steps.

\begin{tabular}{|c|c|c|c|c|c|c|}
\hline Steps & First time & Second time & Third time & Fourth time & Fifth time & Sixth time \\
\hline 1 & 4.44 & 4.86 & 4.92 & 6.43 & 6.61 & 6.44 \\
\hline 2 & 6.97 & 7.21 & 7.24 & 7.79 & 7.99 & 7.88 \\
\hline 3 & 8.17 & 8.34 & 8.38 & 8.9 & 9.12 & 8.98 \\
\hline 4 & 9.76 & 9.88 & 9.68 & 10.34 & 10.49 & 10.46 \\
\hline 5 & 11.27 & 11.36 & 11.31 & 11.74 & 11.91 & 11,87 \\
\hline 6 & 13.13 & 13.14 & 13.02 & 13.49 & 13.79 & $13 / 77$ \\
\hline 7 & 14,97 & 14.92 & 14.77 & 14.2 & 14.49 & 14.46 \\
\hline 8 & 17.04 & 16.84 & 16.71 & 17.07 & $17-49$ & 17.62 \\
\hline 9 & 19.04 & 18.73 & 18.64 & 18.94 & 19.46 & 19.64 \\
\hline 10 & 21.24 & 20.88 & 20.74 & 21.06 & 21.73 & 21.94 \\
\hline 11 & 23.21 & 22.87 & 22.78 & 23.08 & 23.68 & 23.93 \\
\hline 12 & 24.39 & 24.04 & 24.00 & 24.26 & 24.9 & 26.06 \\
\hline 13 & 27.43 & 27.12 & 27.1 & 2121 & 27.89 & 28.04 \\
\hline 14 & 29.6 & 29.33 & 29,36 & 29.43 & 30.22 & 30.23 \\
\hline 15 & 31.67 & 31.37 & 31.42 & 31.64 & 32.3 & 32.29 \\
\hline Foul line & 48.32 & 48.32 & 48.32 & 48.32 & 48.32 & 48.32 \\
\hline Foul distance & -0.15 & -0.15 & -0.23 & -0.17 & 0.15 & 0.24 \\
\hline
\end{tabular}

PToxa1, PToxa2, PToxa3, PToxa4, PToxa5, PToxa6, and PU) is [7-12]. The value of technical and tactical competitive efficiency (its TID PRoxa) is [0-infinity] (its TID is PRoxa1, $\mathrm{PR}$, oxa2, and PEoxa3). To analyze the quality of attack and defense, the analysis mode of volleyball technique and tactics in this study can be analyzed by the following two perspectives: comparative visualization of technical and tactical statistics on both sides of the game and the quality curve of time attack and defense for both sides of the game. The CBA2005-2006 season Shanghai East and Zhejiang Wanma games (January 4, 2006, Yiwu) are an example to discuss the game tactics analysis of the main content and method.

The technical statistics of the game between Shanghai Oriental and Zhejiang Wanma is shown in Figure 3. As shown in Figure 3, although the Shanghai team finally failed in $87: 94$, they were on the low side in the number of free throws (18:33), backcourt times $(40: 49)$, blocked times $(2$ : $6)$, and fouls $(30: 19)$. But in the times of error $(8: 14)$, the number of fast attacks $(4: 0)$, and other indicators, Shanghai was in the upper east. So the good aspects, such as the quick attack, should continue to be carried forward, such as too many fouls (11 times more than the other). It should be carefully analyzed whether the defensive consciousness of a player is in place or not.

In the attack, as shown in Figure 4, Zhejiang cyclones in the first quarter have 10 times the use of the 1 tactical team (Let $\mathrm{Wu}$ Naiqun and Samaki stand on both sides of the waist and put the ball into the inside line and they finish the attack or assists. Give play to the height advantage of internal line foreign aid, Samaki, and the technology of the experience of the forward $\mathrm{Wu}$ Naiqun, the former net strong attack, the latter follow-up). And they get a lot of success. There was the beginning of the start of the 12:5 lead and the end of this section of Shanghai behind at $16: 25$. Shanghai East called a pause, changing the strategy of attack and defense in the east of Shanghai. Foreign aid Oksana avoided opponents edge, to attack distance shots, the defensive front. Samaki had the ball by Wang Ligang then wrapped but also caused the Zhejiang cyclones perimeter defense to weaken accordingly. So the effect is not obvious. Shanghai East, respectively, was 


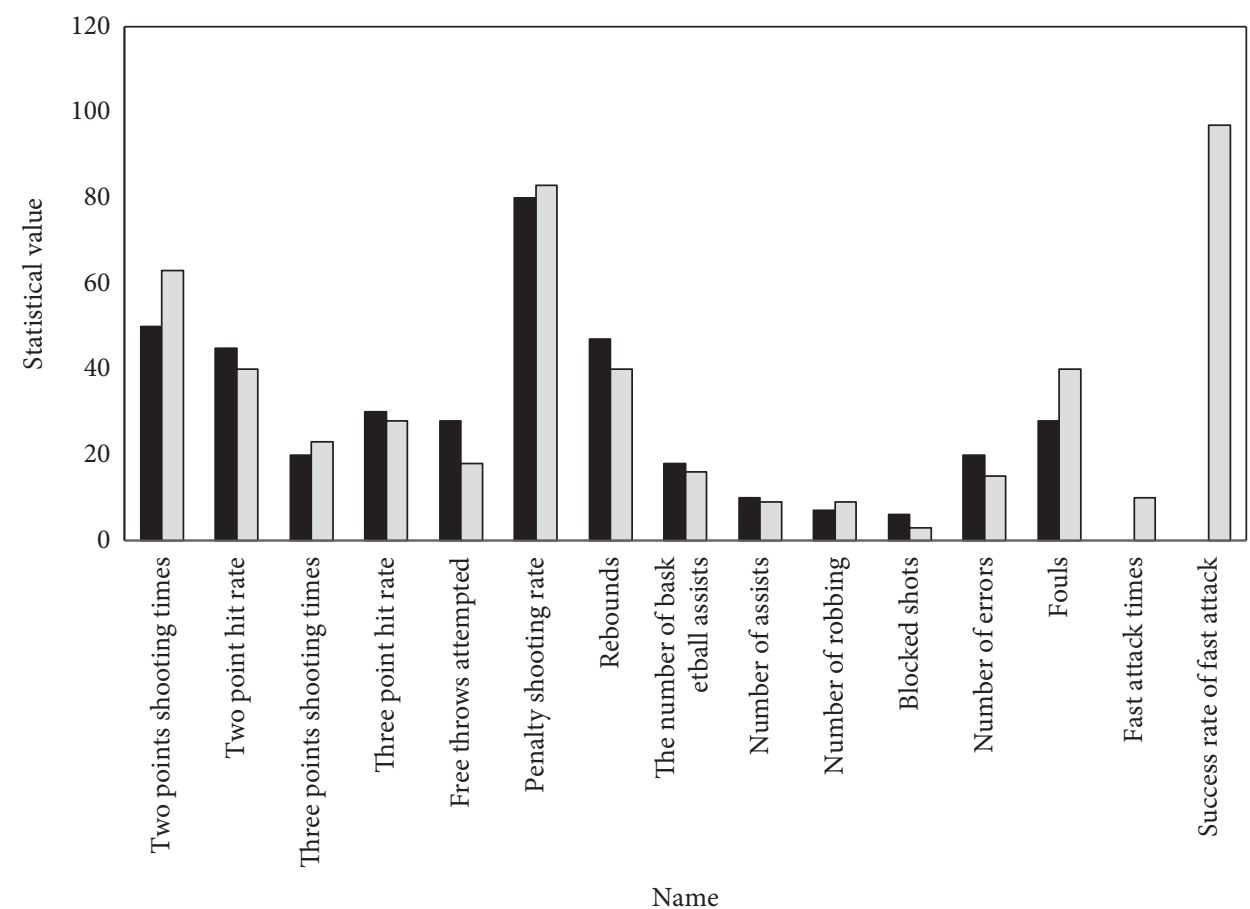

Zhejiang team

$\square$ Shanghai team

Figure 3: Technical statistics of Shanghai Oriental and Zhejiang Wanma game.

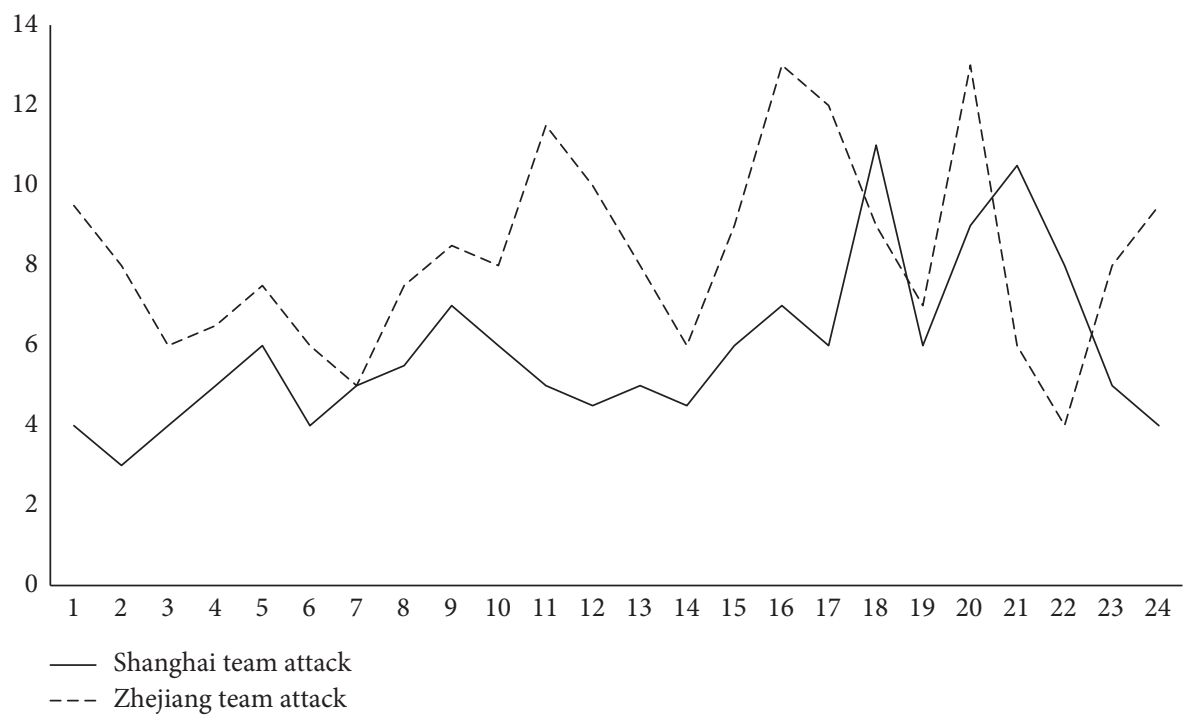

FIgURE 4: Contrast of attack quality in competition.

attacked by Samaki's cap in second and fifth minutes and two turnovers in seventh and tenth minutes. Therefore, there are several inflection points on the curve.

The quality of the attack comparison is provided in Figure 5. As shown in Figure 5, the lead at the start of the third quarter of Zhejiang Wanma bianzhao let Samaki rest, by a small foreign aid ball by Zhang Weigang M. Rudge, in charge. Liu Wei has made 3 mistakes because of his quick strategy for Rudge and the effect of it. Center Zhang Weigang although a larger force, but the pace is 


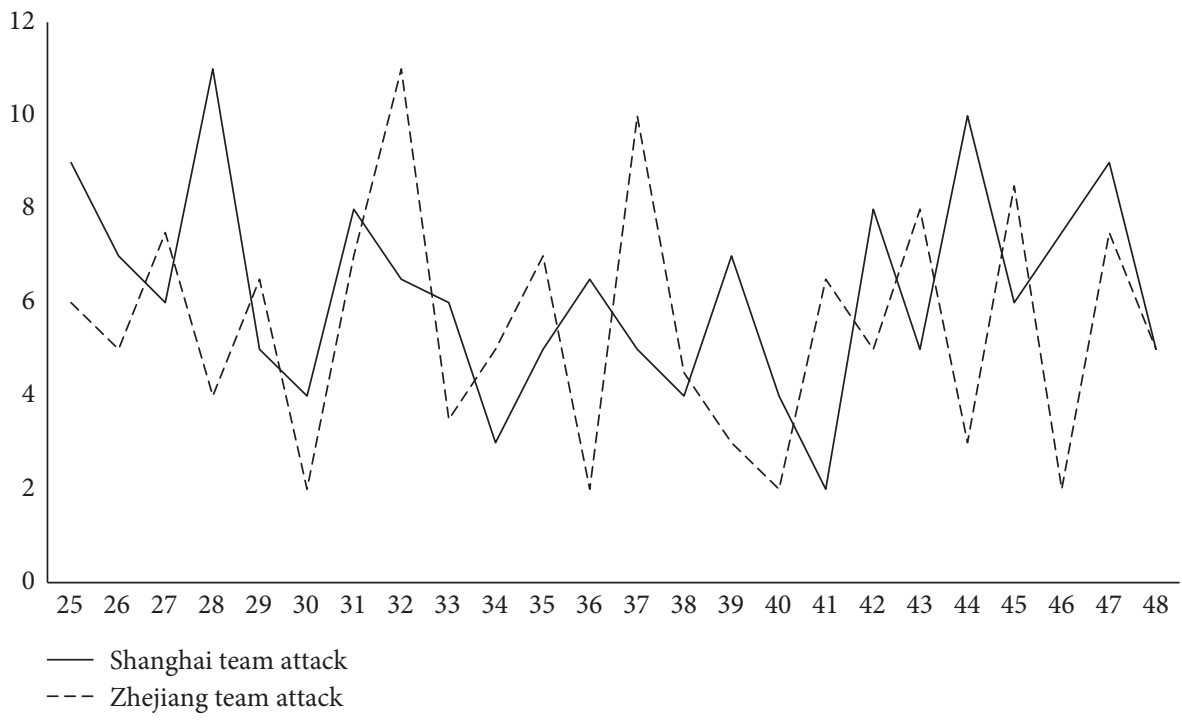

FIGURE 5: Quality attack comparison.

slow, so the poor offensive and defensive Zhejiang cyclones were a disadvantage. In the third section of the Shanghai east to $21: 11$ in the lead, quality of defense graph is Shanghai east line trend compared to the Zhejiang cyclones.

\section{Conclusion}

This article planned to put forward the idea of applying modern data management and data mining to volleyball technology and tactics multimedia data analysis. This article further highlights the idea of designing and developing the multimedia database of volleyball technology and tactics and puts forward the multimedia data analysis method of volleyball technique and tactics based on modern information technology. The proposed model is applied to the data produced at the edges of the systems and thoroughly analyzed. The machine learning algorithm is utilized to process the data and provide a prediction about the strategies of a volleyball match. The classical Apriori algorithm of machine learning is utilized in this research. The classical Apriori algorithm is extended and optimized to overcome the challenges faced by the traditional approach. This paper is mainly aimed at a solution to the lag of the methods and means of volleyball technique and tactics analysis in China. This way can provide intelligence support for coaches and players to enhance their core competitiveness. The proposed optimized Apriori algorithm is implemented and the effectiveness is also highlighted.

\section{Data Availability}

The data used to support the findings of this study are included in the article.

\section{Conflicts of Interest}

The authors declare that they have no conflicts of interest.

\section{References}

[1] H. Li, "Analysis on the construction of sports match prediction model using neural network," Soft Computing, vol. 24, pp. 8343-8353, 2020.

[2] F. Wunderlich and D. Memmert, "Forecasting the outcomes of sports events: a review," European Journal of Sport Science, vol. 12, pp. 1-14, 2020.

[3] B. P. Kelley, C. Klochko, S. Halabi, and D. Siegal, "Datafish multiphase data mining technique to match multiple mutually inclusive independent variables in large PACS databases," Journal of Digital Imaging, vol. 29, no. 3, pp. 331-336, 2015.

[4] P. Wicker and B. Christoph, "Analysis of problems using data mining techniques-findings from sports clubs in Germany," European Journal for Sport and Society, vol. 7, no. 2, pp. 131-140, 2010.

[5] C. Soto Valero, "Predicting win-loss outcomes in MLB regular season games-a comparative study using data mining methods," International Journal of Computer Science in Sport, vol. 15, no. 2, 2016.

[6] X. Yu, F. Jiang, J. Du, and D. Gong, "A cross-domain collaborative filtering algorithm with expanding user and item features via the latent factor space of auxiliary domains," Pattern Recognition, vol. 94, pp. 96-109, 2019.

[7] D. Yang, "Application of data mining technology in the subject tactical teaching of badminton," International Journal of Emerging Technologies in Learning, vol. 13, no. 7, 2018.

[8] G. Raiola, G. Altavilla, C. De Luca, and P. Alfredo Di Tore, "Analysis on some aspects of the service in volleyball," Sport Science, vol. 9, no. 1, pp. 74-77, 2016.

[9] M. Silva, R. Marcelino, D. Lacerda, and P. Vicente João, "Match analysis in volleyball: a systematic review," Montenegrin Journal of Sports Science and Medicine, vol. 5, no. 1, pp. 35-46, 2016.

[10] L. Tianbiao and H. Andreas, "Apriori-based diagnostical analysis of passings in the football game," in Proceeding of the IEEE International Conference on Big Data Analysis (ICBDA), pp. 1-4, IEEE, Hangzhou, China, March 2016.

[11] S. Wenninger, D. Link, and M. Lames, "Performance of machine learning models in application to beach volleyball 
data," International Journal of Computer Science in Sport, vol. 19, no. 1, pp. 24-36, 2020.

[12] J. V. Haaren, H. B. Shitrit, J. Davis, and F. Pascal, "Analyzing volleyball match data from the 2014 world championships using machine learning techniques," in Proceedings of the 22nd ACM SIGKDD International Conference on Knowledge Discovery and Data Mining, pp. 627-634, San Francisco, CA, USA, August 2016.

[13] F. A. Salim, D. B. W. Postma, R. Van Delden et al., "Volleyball action modelling for behavior analysis and interactive multi-modal feedback," in Proceeding of the 15th International Summer Workshop on Multimodal Interfaces, Ankara, Turkey, July 2019.

[14] S. Wenninger, D. Link, and M. Lames, "Data mining in elite beach volleyball-detecting tactical patterns using market basket analysis," International Journal of Computer Science in Sport, vol. 18, no. 2, pp. 1-19, 2019.

[15] X. Yu, J. Yang, and Z. Xie, "Training SVMs on a bound vectors set based on fisher projection," Frontiers of Computer Science, vol. 8, no. 5, pp. 793-806, 2014.

[16] Q.-Y. Li, "Appliance of apriori algorithm on technical-tactics analysis of volleyball," in Proceeding of the International Conference on Virtual Reality and Intelligent Systems (ICVRIS), pp. 164-167, IEEE, Changsha, China, August 2018.

[17] S. Lee, K. Jin, J. Kim, and G. Heo, "Development of a data-mining methodology for spent nuclear fuel forensics," Journal of Radioanalytical and Nuclear Chemistry, vol. 312, no. 3, pp. 495-505, 2017.

[18] X. Yu, Y. Chu, F. Jiang, Y. Guo, and D. Gong, "SVMs classification based two-side cross domain collaborative filtering by inferring intrinsic user and item features," KnowledgeBased Systems, vol. 141, pp. 80-91, 2018.

[19] Z. Haiyun and X. Yizhe, "Sports performance prediction model based on integrated learning algorithm and cloud computing hadoop platform," Microprocessors and Microsystems, vol. 79, Article ID 103322, 2020.

[20] Y. Li, L. Wang, and F. Li, "A data-driven prediction approach for sports team performance and its application to national basketball association," Omega, vol. 98, Article ID 102123, 2019.

[21] A. Dubbs, "Statistics-free sports prediction," Model Assisted Statistics and Applications, vol. 13, no. 2, pp. 173-181, 2018. 